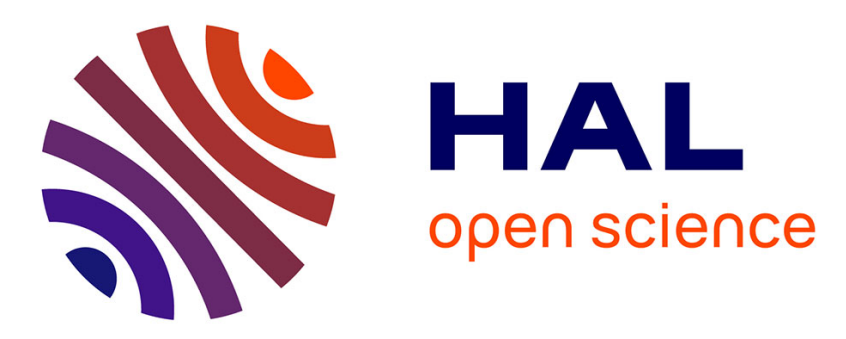

\title{
SAR Image Filtering Based on the Heavy-Tailed Rayleigh Model
}

Alin Achim, Ercan E. Kuruoglu, Josiane Zerubia

\section{To cite this version:}

Alin Achim, Ercan E. Kuruoglu, Josiane Zerubia. SAR Image Filtering Based on the Heavy-Tailed Rayleigh Model. [Research Report] RR-5493, INRIA. 2006, pp.21. inria-00070514

\section{HAL Id: inria-00070514 https://hal.inria.fr/inria-00070514}

Submitted on 19 May 2006

HAL is a multi-disciplinary open access archive for the deposit and dissemination of scientific research documents, whether they are published or not. The documents may come from teaching and research institutions in France or abroad, or from public or private research centers.
L'archive ouverte pluridisciplinaire HAL, est destinée au dépôt et à la diffusion de documents scientifiques de niveau recherche, publiés ou non, émanant des établissements d'enseignement et de recherche français ou étrangers, des laboratoires publics ou privés. 
INSTITUT NATIONAL DE RECHERCHE EN INFORMATIQUE ET EN AUTOMATIQUE

\section{SAR Image Filtering Based on the Heavy-Tailed Rayleigh Model}

Alin Achim — Ercan E. Kuruoglu — Josiane Zerubia

\section{$\mathbf{N}^{\circ} \mathbf{5 4 9 3}$}

February 2005

Thème COG 



\title{
SAR Image Filtering Based on the Heavy-Tailed Rayleigh Model
}

\author{
Alin Achim * , Ercan E. Kuruoglu ${ }^{\dagger}$, Josiane Zerubia ${ }^{\ddagger}$ \\ Thème COG - Systèmes cognitifs \\ Projet Ariana \\ Rapport de recherche $n^{\circ} 5493$ - February 2005 - 21 pages
}

\begin{abstract}
Synthetic aperture radar (SAR) images are inherently affected by a signal dependent noise known as speckle, which is due to the radar wave coherence. In this report, we propose a novel adaptive despeckling filter and derive a maximum a posteriori (MAP) estimator for the radar cross section (RCS). We first employ a logarithmic transformation to change the multiplicative speckle into additive noise. We model the RCS using the recently introduced heavy-tailed Rayleigh density function, which was derived based on the assumption that the real and imaginary parts of the received complex signal are best described using the alpha-stable family of distribution. We estimate model parameters from noisy observations by means of second-kind statistics theory, which relies on the Mellin transform. Finally, we compare our proposed algorithm with several classical speckle filters applied on actual SAR images. Experimental results show that the homomorphic MAP filter based on the heavy-tailed Rayleigh prior for the RCS is among the best for speckle removal.
\end{abstract}

Key-words: Synthetic Aperture Radar, MAP estimation, alpha-stable distributions, heavy-tailed Rayleigh model, Mellin transform

\footnotetext{
* Signal Processing Group, Department of Electrical \& Electronic Engineering, University of Bristol, BS8 1UB, Bristol (UK), e-mail: Alin.Achim@bristol.ac.uk

† Signals and Images Laboratory, ISTI-CNR, Area della Ricerca CNR di Pisa, via G. Moruzzi 1, I-56124, Pisa (Italy), e-mail: Ercan.Kuruoglu@isti.cnr.it

$\ddagger$ Projet Ariana, UR INRIA Sophia Antipolis, 2004 Route des Lucioles, B.P. 93, FR-06902, Sophia Antipolis Cedex (France), e-mail: Josiane.Zerubia@inria.fr
} 


\section{Filtrage d' Images Radar RSO Fondé sur le Modèle de Rayleigh à Queue Lourde}

Résumé : Les images issues d'un radar à synthèse d'ouverture (RSO) sont affectées de manière inhérente par un bruit dépendant du signal, généralement connu sous le nom de bruit de chatoiement et qui est dû à la cohérence de l'onde radar. Dans ce rapport, nous proposons un nouveau filtre adaptatif pour débruiter les images RSO et nous déduisons un estimateur du maximum a posteriori (MAP) pour la section efficace du diagramme de gain en radar. On utilise d'abord une transformée logarithmique afin de changer le bruit multiplicatif en bruit additif. Nous modélisons la section efficace à l'aide d'une densité de probabilité récemment introduite - la densité de Rayleigh à queue lourde, qui a été obtenue en supposant que les parties réelles et imaginaires du signal complexe reçu peuvent être mieux caractérisées à l'aide de la famille des distributions alpha-stables. Nous estimons les paramètres du modèle à partir d'observations bruitées en faisant appel à la théorie statistique de deuxième espèce qui est fondée sur la transformée de Mellin. Enfin, nous faisons la comparaison entre la méthode que nous proposons et d'autres filtres classiques pour le débruitage d'images RSO. Nos résultats expérimentaux démontrent que le filtre MAP homomorphique fondé sur le modèle de Rayleigh à queue lourde est parmi les meilleurs pour enlever le bruit de chatoiement.

Mots-clés : Radar à Synthèse d'Ouverture (RSO), estimation MAP, distributions alphastables, modèle de Rayleigh à queue lourde, transformée de Mellin 


\section{Contents}

1 Introduction 4

2 Statistical modeling of SAR images 5

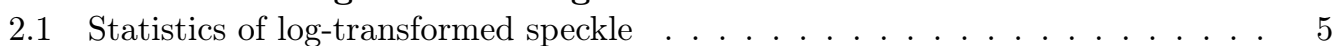

2.1 .1 Intensity Image . . . . . . . . . . . . . . . . . . . 6

2.1 .2 Amplitude Image . . . . . . . . . . . . . . . . . . . . . . . . . 7

2.2 The generalized Rayleigh model . . . . . . . . . . . . . . . . . . . . . 8

2.2.1 Symmetric Alpha-Stable Distributions . . . . . . . . . . . . . . 8

2.2 .2 A Heavy-Tailed Rayleigh model . . . . . . . . . . . . . . . . . 8

3 Adaptive MAP filtering of speckle noise $\quad 9$

3.1 Parameter estimation using Mellin transform . . . . . . . . . . . . . . . . 11

3.1 .1 Mellin transform . . . . . . . . . . . . . . . . . . . . 11

3.1.2 Log-moment estimation of the generalized Rayleigh model . . . . . . . 12

4 Experimental Results $\quad \mathbf{1 4}$

4.1 Synthetic Data Examples . . . . . . . . . . . . . . . . . . . . . 14

4.2 Real SAR Imagery Examples . . . . . . . . . . . . . . . . . . . 15

$\begin{array}{llr}5 & \text { Conclusions } & 18\end{array}$

6 Acknowledgement $\quad 18$

$\mathrm{RR} \mathrm{n}^{\circ} 5493$ 


\section{Introduction}

Over the last couple of decades, there has been a growing interest in synthetic aperture radar (SAR) imaging on account of its importance in a variety of applications such as remote sensing for mapping, search-and-rescue, mine detection, and target recognition. SAR is an active coherent microwave imaging method that utilizes the motion of a radar physical aperture antenna mounted on a platform, such as an aircraft or a satellite, to synthesize the effect of a long aperture in order to achieve the high resolution of a virtual focused array system with a much larger aperture [1]. Modern airborne and satellite-borne SAR systems are capable of producing high-quality pictures of the earth's surface while avoiding some of the shortcomings of other forms of remote imaging systems. Specifically, SAR imaging systems overcome the night-time limitations of optical cameras and the cloud-cover limitations of infrared imagers.

A major issue in SAR imagery is that basic textures are generally affected by multiplicative speckle noise [2]. Speckle noise is a consequence of image formation under coherent radiation. It is not truly a noise in the typical engineering sense, since its texture often carries useful information about the scene being imaged. However, the presence of speckle is generally considered undesirable since it damages radiometric resolution and it affects the tasks of human interpretation and scene analysis. Thus, it appears sensible to reduce speckle in SAR images, provided that the structural features and textural information are not lost.

Many adaptive filters for SAR image denoising have been proposed in the past. The simplest approaches to speckle reduction are based on temporal averaging [2,3], median filtering, and Wiener filtering. The classical Wiener filter, which utilizes the second order statistics of the Fourier decomposition, is not adequate for removing speckle since it is designed mainly for additive noise suppression. To address the multiplicative nature of speckle noise, Jain developed a homomorphic approach, which by taking the logarithm of the image, converts the multiplicative into additive noise, and consequently applies the Wiener filter [4]. The Frost filter was designed as an adaptive Wiener filter that assumed an autoregressive (AR) exponential model for the scene reflectivity [5]. Kuan considered a multiplicative speckle model and designed a linear filter based on the minimum meansquare error (MMSE) criterion, optimal when both the scene and the detected intensities are Gaussian distributed [6]. The Lee MMSE filter was a particular case of the Kuan filter based on a linear approximation made for the multiplicative noise model [7]. A twodimensional Kalman filter was developed by Sadjadi and Bannour under the modeling of the image as a Markov field satisfying a causal AR model [8]. For the case of co-registered SAR images, Bruniquel and Lopes [9] developed a speckle reduction method based on pixelto-pixel summation. The Gamma MAP filter was based on a Bayesian analysis of the image statistics where both radar cross section (RCS) and speckle noise follow a Gamma distribution [10]. Finally, a number of recently developed filters were based on wavelet transform $[11,12,13,14]$.

Although wavelet-based filters have been shown to generally outperform those based on single scale representations of signals, the overall performance of the former could be improved by adopting better statistical models for the later. Indeed, in [14] Solbo and 
Eltoft developed the $\Gamma-W M A P$ filter, a filter based on the same statistical assumptions as for the Gamma MAP but implemented in the wavelet domain. Specifically, they proposed the use of normal inverse Gaussian (NIG) distributions as a statistical model for the wavelet coefficients of both the reflectance image and the noise. Then, in order to implement a MAP processor, they estimate the parameters of the NIG distributions by relating the cumulants in the wavelet domain to the cumulants of the assumed Gamma distributions in the pixel domain. We believe, their methodology could further be improved by making use of models that better characterize the statistical properties of the RCS.

In this report we propose the use of an alternative RCS model for designing a speckle removal filter. Thus, we employ the heavy-tailed Rayleigh distribution [15] that was shown to be well justified by the physics of the radar wave scattering. Specifically, the model was developed based on the observation that the real and imaginary parts of the received complex signal can be accurately modelled using the symmetric alpha-stable family of distribution $[16,17]$. The generalized Gaussian Rayleigh model $[18,19]$ arising from the assumption that the real and imaginary parts of the backscattered SAR signal are distributed according to a generalized Gaussian distribution, could have been an equally appropriate choice. Under the assumption of a multiplicative speckle noise model, we first employ a logarithmic transformation in order to change the noise into an additive one and to differentiate its characteristics from the signal characteristics. Then, the general MAP solution for the resulting model is derived and the model parameter estimation is presented. The proposed estimation method is based on the second-kind statistic theory employing Mellin's transform [20] as recently proposed by Nicolas and co-authors [21, 22].

The report is organized as follows. In Section 2 we discuss the statistical properties of SAR images, as well as those of log-transformed images. In Section 3, we present the design of our MAP estimator based on the heavy-tailed Rayleigh signal model, which includes a novel parameter estimation method based on Mellin transform. In Section 4, we evaluate the performance of our proposed filter and we compare it with existing speckle removal methods. Finally, in Section 5 we conclude the report with a short summary.

\section{Statistical modeling of SAR images}

Parametric Bayesian processing presupposes proper modeling for the prior probability density function (pdf) of both the radar cross section and speckle noise. In this section we briefly review the statistical properties of speckle and we describe the model used for the RCS.

\subsection{Statistics of log-transformed speckle}

Speckle noise affects all coherent imaging systems including laser, SAR imagery, and ultrasound. Speckle may appear distinct in different imaging systems but it is always manifested in a granular pattern due to image formation under coherent waves.

$\mathrm{RR} \mathrm{n}^{\circ} 5493$ 
The statistical properties of speckle noise were studied by Goodman [2]. He has shown that, if the number of scatterers per resolution cell is large, a fully developed speckle pattern can be modeled as the magnitude of a complex Gaussian field with i.i.d. real and imaginary components. Realistic speckle noise models have been developed in the past and include the K-distribution [23], $\mathcal{G}$-distribution [24], log-normal distribution [13, 25], and correlated speckle pattern [23, 26]. A general model for speckle noise proposed by Jain [4] is constantly employed when one is concerned with the implementation of a homomorphic filter. Specifically, if we denote by $y(u, v)$ a noisy observation (i.e., the recorded SAR image envelope) of the two-dimensional function $x(u, v)$ (i.e., the noise-free SAR image that has to be recovered) and by $\eta(u, v)$ and $\xi(u, v)$ the corrupting multiplicative and additive speckle noise components, respectively, one can write:

$$
y(u, v)=x(u, v) \cdot \eta(u, v)+\xi(u, v), \quad(u, v) \in \mathbf{Z}^{2}
$$

Generally, the effect of the additive component of the speckle in SAR images is less significant than the effect of the multiplicative component. Thus, ignoring the term $\xi(u, v)$, one can rewrite (1) as

$$
y(u, v)=x(u, v) \cdot \eta(u, v)
$$

To transform the multiplicative noise model into an additive one, we apply the logarithmic function on both sides of (2):

$$
\log y(u, v)=\log x(u, v)+\log \eta(u, v) .
$$

Expression (2) can be rewritten as

$$
Y(u, v)=X(u, v)+N(u, v)
$$

where $Y(\cdot), X(\cdot)$, and $N(\cdot)$ are the logarithms of $y(\cdot), x(\cdot)$, and $\eta(\cdot)$, respectively. In sections 2.1.1 and 2.1.2, we briefly describe the statistical properties of speckle noise in both the original and the logarithmic transform domain.

\subsubsection{Intensity Image}

The statistical properties of speckle noise have been first studied by Arsenault and April [27] who have shown that when the image intensity is logarithmically transformed, speckle is approximately Gaussian additive noise, and it tends to a normal probability much faster than the distribution of the intensity image. Xie et al. employ a distance between cumulative distributions to measure the deviation of the log-transformed speckle from Gaussianity [28]. They confirm the result in [27] and show that even for the amplitude image, although the log-transformed speckle tends to a Gaussian pdf slightly slower than the original speckle noise, the former is still statistically very close to the Gaussian PDF. Nevertheless, closed form analytical expressions can be obtained for the logarithmically transformed speckle noise starting from the physics of wave scattering. For a SAR image representing an average of 
L looks in intensity format, the speckle noise random variable $\eta$ in (2) follows a Gamma distribution with unit mean and variance $1 / L$. Its pdf can be written as

$$
p_{I}(\eta)=\frac{L^{L} \eta^{L-1} e^{-L \eta}}{\Gamma(L)}
$$

For reasons that will become obvious within the next sections, we also provide here the first and second orders log-cumulants of a Gamma distribution

$$
\begin{array}{r}
\tilde{k}_{I(1)}=\Psi(L)-\log (L) \\
\tilde{k}_{I(2)}=\Psi(1, L)
\end{array}
$$

where $\Psi$ is the Digamma function and $\Psi(r, L)$ is the Polygamma function, i.e. the r-th derivative of the Digamma function. Having in mind that $p(\eta) d \eta=p(N) d N$, one can readily obtain the pdf of the random variable $N=\log \eta$

$$
p_{I}(N)=\frac{L^{L} e^{N L} e^{-L e^{N}}}{\Gamma(L)}
$$

\subsubsection{Amplitude Image}

For an amplitude image, the pdf and the expressions for the log-cumulants can be obtained from the ones corresponding to the intensity image by recalling that [21]

$$
\begin{aligned}
p_{A}(x) & =2 x p_{I}\left(x^{2}\right) \\
\tilde{k}_{A(r)} & =\left(\frac{1}{2}\right)^{r} \tilde{k}_{I(r)}
\end{aligned}
$$

Consequently, using the above expressions with (5) and (6) one obtain respectively

$$
p_{A}(\eta)=\frac{2 L^{L} \eta^{2 L-1} e^{-L \eta^{2}}}{\Gamma(L)}
$$

which is basically the Nakagami distribution and

$$
\begin{array}{r}
\tilde{k}_{A(1)}=\frac{1}{2}(\Psi(L)-\log (L)) \\
\tilde{k}_{A(2)}=\frac{1}{4} \Psi(1, L)
\end{array}
$$

Finally, the pdf of the random variable $N=\log (\eta)$ for amplitude images is given by

$$
p_{A}(N)=\frac{2 L^{L} e^{2 N L} e^{-L e^{2 N}}}{\Gamma(L)}
$$

$\mathrm{RR} \mathrm{n}^{\circ} 5493$ 


\subsection{The generalized Rayleigh model}

The SAR image formation theory has been long time dominated by the assumption of Gaussianity for the real and imaginary parts of the received complex signals. Based on this assumption, the detected amplitude SAR images can be modeled by a Rayleigh distribution. However, as we will show in this section, invoking a generalized version of the central limit theorem, the assumption of Gaussianity can be replaced by an assumption of "alpha-stability" resulting in a more powerful model for the detected amplitude pdf. In the following we provide a brief, necessary overview of the alpha-stable statistical model on which the generalized Rayleigh pdf is actually based.

\subsubsection{Symmetric Alpha-Stable Distributions}

The appeal of $S \alpha S$ distributions as a statistical model for signals derives from two main theoretical reasons. First, stable random variables satisfy the stability property which states that linear combinations of jointly stable variables are indeed stable. The word stable is used because the shape of the distribution is unchanged (or stable) under such linear combinations. Second, stable processes arise as limiting processes of sums of independent identically distributed (i.i.d.) random variables via the generalized central limit theorem. Actually, the only possible non-trivial limit of normalized sums of i.i.d. terms is stable.

The $S \alpha S$ distribution lacks a compact analytical expression for its probability density function (pdf). Consequently, it is most conveniently represented by its characteristic function

$$
\varphi(\omega)=\exp \left(\jmath \delta \omega-\gamma|\omega|^{\alpha}\right)
$$

where $\alpha$ is the characteristic exponent, taking values $0<\alpha \leq 2, \delta(-\infty<\delta<\infty)$ is the location parameter, and $\gamma(\gamma>0)$ is the dispersion of the distribution. For values of $\alpha$ in the interval $(1,2]$, the location parameter $\delta$ corresponds to the mean of the $S \alpha S$ distribution, while for $0<\alpha \leq 1, \delta$ corresponds to its median. The dispersion parameter $\gamma$ determines the spread of the distribution around its location parameter $\delta$, similar to the variance of the Gaussian distribution.

The characteristic exponent $\alpha$ is the most important parameter of the $S \alpha S$ distribution and it determines the shape of the distribution. The smaller the characteristic exponent $\alpha$ is, the heavier the tails of the $S \alpha S$ density. This implies that random variables following $S \alpha S$ distributions with small characteristic exponents are highly impulsive. One consequence of heavy tails is that only moments of order less than $\alpha$ exist for the non-Gaussian alphastable family members. As a result, stable laws have infinite variance. Gaussian processes are stable processes with $\alpha=2$ while Cauchy processes result when $\alpha=1$.

\subsubsection{A Heavy-Tailed Rayleigh model}

Kuruoglu and Zerubia [15] assumed that the real and imaginary parts of the received SAR signal are jointly $S \alpha S$. Consequently, they derived the following integral equation for the 
amplitude pdf of SAR images, which they called the heavy-tailed Rayleigh distribution:

$$
p(x)=x \int_{0}^{\infty} u \exp \left(-\gamma u^{\alpha}\right) J_{0}(u x) d u
$$

where $J_{0}$ is the zeroth order Bessel function of the first kind.

It is important to note at this point, that by considering the special case $\alpha=2$, we obtain

$$
p(x)=\frac{x}{2 \gamma} \exp \left(-\frac{x^{2}}{4 \gamma}\right)
$$

which is basically the classical Rayleigh distribution as expected since for $\alpha=2$ the $S \alpha S$ distribution reduces to Gaussian. In fact, in [15] the authors have shown that the heavytailed Rayleigh distribution can be expressed as a mixture of Rayleighs. Also, by taking $\alpha=1$ in (14), one obtains the following pdf, which we will refer to as the Cauchy-Rayleigh model

$$
p(x)=\frac{x \gamma}{\left(x^{2}+\gamma^{2}\right)^{3 / 2}}
$$

In Fig. 1 we show the tail behavior of several heavy-tailed Rayleigh densities including the particular cases corresponding to the Cauchy and the Gaussian distributions. For the case of images in intensity format the pdf can be readily obtained from (14) recalling that $p_{A}(x)=2 x p_{I}\left(x^{2}\right)$. Thus, one obtain

$$
p_{I}(x)=\frac{1}{2} \int_{0}^{\infty} u \exp \left(-\gamma u^{\alpha}\right) J_{0}(u \sqrt{x}) d u
$$

In our further developments we will employ a homomorphic transformation in order to transform the multiplicative speckle noise into an additive one. Consequently, let us also provide here the logarithmic domain pdfs corresponding to the heavy-tailed Rayleigh model in both amplitude and intensity formats respectively

$$
\begin{aligned}
& p_{A}(X)=e^{2 X} \int_{0}^{\infty} u \exp \left(-\gamma u^{\alpha}\right) J_{0}\left(u e^{X}\right) d u \\
& p_{I}(X)=\frac{e^{X}}{2} \int_{0}^{\infty} u \exp \left(-\gamma u^{\alpha}\right) J_{0}\left(u e^{\frac{X}{2}}\right) d u
\end{aligned}
$$

where $X=\ln x$.

\section{Adaptive MAP filtering of speckle noise}

After applying a logarithmic transformation to the original data we get an image represented as the sum of the transformations of the signal and of the noise:

$$
Y=X+N
$$

$\mathrm{RR} \mathrm{n}^{\circ} 5493$ 


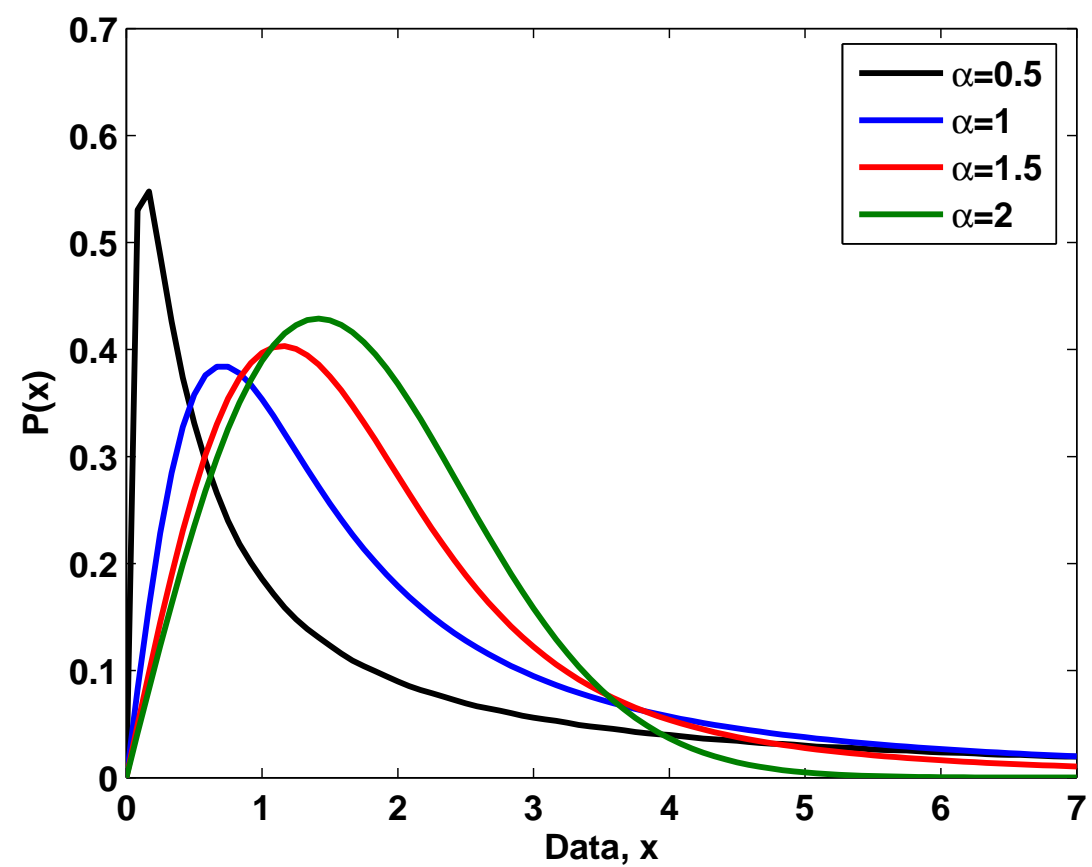

Figure 1: Generalized Rayleigh probability density functions for different values of the characteristic exponent $\alpha$. The dispersion parameter is kept constant at $\gamma=1$.

The MAP estimator of $X$ given the noisy observation $Y$ is:

$$
\hat{X}(Y)=\arg \max _{X} P_{X \mid Y}(X \mid Y)
$$

Bayes' theorem gives the a posteriori PDF of $X$ based on the measured data:

$$
P_{X \mid Y}(X \mid Y)=\frac{P_{Y \mid X}(Y \mid X) P_{X}(X)}{P_{Y}(Y)},
$$

where $P_{X}(X)$ is the prior $\mathrm{PDF}$ of the signal component of the measurements and $P_{Y \mid X}(Y \mid X)$ is the likelihood function. Substituting (22) in (21), we get:

$$
\hat{X}(Y)=\arg \max _{X} P_{Y \mid X}(Y \mid X) P_{X}(X)=\arg \max _{X} P_{N}(Y-X) P_{X}(X)
$$




$$
=\arg \max _{X} P_{N}(N) P_{X}(X)
$$

In the above equation we use a heavy-tailed Rayleigh model for the signal component, while we use a Gamma or Nakagami model for the noise component depending on whether the image to be filtered is in intensity or in amplitude format, respectively. Naturally, in order for the processor in Eq. (23) to be of any practical use, one should be able to estimate the parameters $\alpha_{X}$ and $\gamma_{X}$ of the signal from the observed data. In the next subsection we derive parameter estimation methods for the generalized Rayleigh pdf based on second-kind cumulants.

\subsection{Parameter estimation using Mellin transform}

In order to develop a robust MAP filter for SAR images, the heavy-tailed Rayleigh model parameters characterizing the RCS need to be accurately estimated from noisy observations. Following the arguments in [29] and [30], Nicolas has recently proposed the use of Mellin transform as a powerful tool for deriving novel parameter estimation methods based on logcumulants for the case of multiplicative noise contamination [21, 22]. In the following, we briefly review the Mellin transform and its main properties that we used in our derivations.

\subsubsection{Mellin transform}

Let $f$ be a function defined over $\Re^{+}$. The integral transform defined by

$$
\Phi(s)=\mathbf{M}[f(u)](s)=\int_{0}^{+\infty} u^{s-1} f(u) d u
$$

is called the Mellin transform of $f$. The inverse transform is given by

$$
f(u)=\mathbf{M}^{-1}[\Phi(s)](u)=\frac{1}{2 \pi j} \int_{c-j \infty}^{c+j \infty} u^{-s} \Phi(s) d s
$$

The transform $\Phi(s)$ exists if the integral $\int_{0}^{+\infty}|f(x)| x^{k-1} d x$ is bounded for some $k>0$, in which case the inverse $\mathrm{f}(\mathrm{u})$ exists with $\mathrm{c}>\mathrm{k}$. The functions $\Phi(s)$ and $f(u)$ are called a Mellin transform pair, and either can be computed if the other is known.

By analogy with the way in which common statistics are deducted based on Fourier Transform, the following second-kind statistic functions can be defined, based on Mellin Transform [21, 22]

- Second-kind first characteristic function

$$
\Phi(s)=\int_{0}^{+\infty} x^{s-1} p(x) d x
$$

- Second-kind second characteristic function

$$
\Psi(s)=\log (\Phi(s))
$$

$\mathrm{RR} \mathrm{n}^{\circ} 5493$ 
- $r^{\text {th }}$ order second-kind moments

$$
\tilde{m}_{r}=\left.\frac{d^{r} \Phi(s)}{d s^{r}}\right|_{s=1}=\int_{0}^{+\infty}(\log x)^{r} p(x) d x
$$

- $r^{\text {th }}$ order second-kind cumulants

$$
\tilde{k}_{r}=\left.\frac{d^{r} \Psi(s)}{d s^{r}}\right|_{s=1}
$$

The first two second-kind cumulants can be estimated empirically from $\mathrm{N}$ samples $y_{i}$ as follows

$$
\begin{array}{r}
\hat{\tilde{k}}_{1}=\frac{1}{N} \sum_{i=1}^{N}\left[\log \left(y_{i}\right)\right] \\
\hat{\tilde{k}}_{2}=\frac{1}{N} \sum_{i=1}^{N}\left[\left(\log \left(y_{i}\right)-\hat{\tilde{k}}_{1}\right)^{2}\right]
\end{array}
$$

- Finally, for two functions $f$ and $g$, Mellin's convolution is defined over the interval $[0, \infty]$ as

$$
(f \hat{*} g)(y)=\int_{0}^{+\infty} f(x) g\left(\frac{y}{x}\right) \frac{d x}{x}=\int_{0}^{+\infty} f\left(\frac{y}{x}\right) g(x) \frac{d x}{x}
$$

\subsubsection{Log-moment estimation of the generalized Rayleigh model}

By plugging the expression of the heavy-tailed Rayleigh pdf given by (14) into (26) and after some straightforward manipulations, details of which can be found in [15], one gets

$$
\Phi(s)=\frac{2^{s} \Gamma\left(\frac{s+1}{2}\right) \gamma^{\frac{s-1}{\alpha}} \Gamma\left(\frac{1-s}{\alpha}\right)}{\Gamma\left(\frac{1-s}{2}\right) \alpha}
$$

which is the second-kind first characteristic function of the heavy-tailed Rayleigh density. Kuruoglu and Zerubia $[15,31]$ used this expression for two different values of $s$ and subsequently solved the resulting system in order to get estimates of the parameters $\alpha$ and $\gamma$. However, here we are interested in deriving estimates of the model parameters in the case of multiplicative noise contamination. Consequently, we settled by plugging the above expression in (27) and subsequently in (29), thus obtaining the following results for the second-kind cumulants of the model

$$
\begin{array}{r}
\tilde{k}_{A(1)}=-\psi(1) \frac{1-\alpha}{\alpha}+\log \left(2 \gamma^{\frac{1}{\alpha}}\right) \\
\tilde{k}_{A(2)}=\frac{\psi(1,1)}{\alpha^{2}}
\end{array}
$$


For the case of intensity images, using the same methodology as above one can obtain the following expressions for the log-cumulants

$$
\begin{array}{r}
\tilde{k}_{I(1)}=-2 \psi(1) \frac{1-\alpha}{\alpha}+\log \left(4 \gamma^{\frac{2}{\alpha}}\right) \\
\tilde{k}_{I(2)}=4 \frac{\psi(1,1)}{\alpha^{2}}
\end{array}
$$

Using either of the above systems of two equations one can readily solve for the parameters $\alpha$ and $\gamma$ of the heavy-tailed Rayleigh distribution. Remember however that our measurement is a mixture of heavy-tailed Rayleigh signal and Nakagami (or Gamma) distributed speckle noise. Under the multiplicative speckle noise model (2), if we denote by $p_{y}(y), p_{x}(x)$, and $p_{\eta}(\eta)$ the pdfs of $y, x, \eta$ respectively, it can be shown that the pdf of $y$ is given in fact by the Mellin convolution between the pdfs of $x$ and $\eta$, since

$$
p_{y}(y)=\int_{0}^{+\infty} p_{y \mid x}(y \mid x) p_{x}(x) d x=\int_{0}^{+\infty} p_{\eta}\left(\frac{y}{x}\right) p_{x}(x) \frac{d x}{x}=p_{\eta} \hat{*} p_{x}
$$

Consequently, the second-kind cumulant of any order of $y$ is given by the sum of the secondkind cumulants of the same order of $x$ and $\eta[21]$

$$
\tilde{k}_{y(r)}=\tilde{k}_{x(r)}+\tilde{k}_{\eta(r)}
$$

Using expressions (6) and (34) in the above equation together with the empirical logcumulants in (30) we obtain the following estimates for the parameters of the heavy-tailed Rayleigh model (17) mixed with Gamma distributed speckle noise

$$
\begin{array}{r}
\hat{\alpha}=2 \cdot \sqrt{\frac{\psi(1,1)}{\hat{\tilde{k}}_{(2)}-\psi(1, L)}} \\
\hat{\gamma}=\left[\frac{\exp \left(\hat{\tilde{k}}_{(1)}+2 \psi(1) \frac{1-\alpha}{\alpha}-\psi(L)+\log (L)\right.}{4}\right]^{\alpha / 2}
\end{array}
$$

Similarly, for the case of amplitude images, using expressions (11) and (33) in (36) with the empirical log-cumulants in (30) we get

$$
\begin{array}{r}
\hat{\alpha}=\sqrt{\frac{\psi(1,1)}{\hat{\tilde{k}}_{(2)}-\frac{1}{4} \cdot \psi(1, L)}} \\
\hat{\gamma}=\left[\frac{\exp \left(\hat{\tilde{k}}_{(1)}+\psi(1) \frac{1-\alpha}{\alpha}-\frac{1}{2}(\psi(L)-\log (L))\right.}{2}\right]^{\alpha}
\end{array}
$$

$\mathrm{RR} \mathrm{n}^{\circ} 5493$ 


\section{Experimental Results}

In this section, we present simulation results obtained by processing several test SAR images using our proposed MAP speckle filter based on the heavy-tailed Rayleigh prior. We compared the results of our approach with those obtained using other classical speckle filters including the median, the homomorphic Wiener, the Lee, and the $\Gamma$-MAP filter.

\subsection{Synthetic Data Examples}

We started by first degrading an original "speckleless" image with synthetic speckle in amplitude format. For this purpose, an aerial image was chosen due to its identical content with real SAR images. This image was obtained by cropping the "westaerialconcorde" image that can be found in Matlab's Image Processing Toolbox. In our experiments, we considered four different levels of simulated speckle noise corresponding to $L=1,3,9$, and 12 (cf. eq. 10).

In order to assess the quality of our proposed filter we computed two different measures based on the original and the denoised data. A common way to evaluate the noise suppression in case of multiplicative contamination is to calculate the signal-to-mean squared error (S/MSE) ratio, defined as [25]:

$$
S / M S E=10 \log _{10}\left(\sum_{i=1}^{K} S_{i}^{2} / \sum_{i=1}^{K}\left(\hat{S}_{i}-S_{i}\right)^{2}\right)
$$

where $S$ is the original image, $\hat{S}$ is the denoised image, and $K$ is the image size. This measure corresponds to the classical SNR in the case of additive noise.

In addition to the above quantitative performance measure, we also consider a qualitative measure for edge preservation. Specifically, we used a parameter $\beta$ originally defined in [32]:

$$
\beta=\frac{\Gamma(\Delta S-\overline{\Delta S}, \widehat{\Delta S}-\overline{\overline{\Delta S}})}{\sqrt{\Gamma(\Delta S-\overline{\Delta S}, \Delta S-\overline{\Delta S}) \cdot \Gamma(\widehat{\Delta S}-\overline{\overline{\Delta S}}, \widehat{\Delta S}-\overline{\overline{\Delta S}})}}
$$

where $\Delta S$ and $\widehat{\Delta S}$ are the high-pass filtered versions of $S$ and $\hat{S}$ respectively, obtained with a $3 \times 3$-pixel standard approximation of the Laplacian operator, and

$$
\Gamma\left(S_{1}, S_{2}\right)=\sum_{i=1}^{K} S_{1_{i}} \cdot S_{2_{i}} .
$$

The correlation measure, $\beta$ should be close to unity for an optimal effect of edge preservation. For this experiment we chose to compare our filter with the median, the homomorphic Wiener and the Lee filters. All these filters were implemented adaptively using a square shaped sliding window of size $7 \times 7$. The obtained values of $S / M S E$, and $\beta$ for all methods applied to our test image are given in Table 1. From the table it can be seen that, in most situations, our proposed filter exhibits the best performance according to both metrics. 
Table 1: Image enhancement measures obtained by four denoising methods applied on the "aerial" image. Four levels of noise are considered corresponding to ENL=1, 3, 9, and 12. The $S / M S E$ of each despeckled image is given in $d B$.

\begin{tabular}{|l||c|c||c|c||c|c||c|c|}
\hline \multicolumn{1}{|c||}{} & \multicolumn{2}{c||}{ ENL $=1$} & \multicolumn{2}{c||}{ ENL $=3$} & \multicolumn{2}{c||}{ ENL $=9$} & \multicolumn{2}{c|}{ ENL $=12$} \\
\hline Method & $S / M S E$ & $\beta$ & $S / M S E$ & $\beta$ & $S / M S E$ & $\beta$ & $S / M S E$ & $\beta$ \\
\hline \hline Median & 14.53 & 0.2421 & 15.52 & 0.3309 & 15.85 & 0.3639 & 15.88 & 0.3677 \\
\hline Wiener & 14.23 & 0.3187 & 15.27 & 0.3812 & 15.56 & 0.4097 & 15.60 & 0.4150 \\
\hline Lee & 14.69 & 0.2118 & 15.35 & 0.3540 & 16.64 & 0.5417 & 17.28 & 0.6186 \\
\hline proposed & 15.86 & 0.2556 & 16.83 & 0.3541 & 18.00 & 0.5833 & 18.68 & 0.6471 \\
\hline
\end{tabular}

Figure 2 shows a representative result from the processing of the aerial test image. The image in Figure 2(b) was obtained by degrading the original test image (2(a)) with Nakagami distributed speckle noise (cf. eq. 10) with $L=3$ looks. From the figure it can be seen that all the tested filters achieved a good speckle surpressing performance. However, clearly our homomorphic MAP filter based on the heavy-tailed Rayleigh signal prior did the best job in preserving the structural features that can be observed in the original image.

\subsection{Real SAR Imagery Examples}

In order to further study the merit of the proposed generalized Rayleigh-based MAP processor, we also chose noisy SAR images, we applied the algorithm without artificially adding noise, and we visually evaluated the denoised images. The test image, shown in Figure 3(a), depicts mainly a rural scene, but one can also see the city of Bourges and some other villages, forests, an airport, a river and many roads. The image is in 4-looks, amplitude format and was acquired in April 1993 by ERS.

For visually assessing the quality of filtered images, we show results obtained using the ГMAP filter (Figures 3(b)) and the generalized Rayleigh-based MAP (Figures 3(c)). Although qualitative evaluation in this case is highly subjective, i.e., no universal quality measure for filtered SAR data exists, we also chose to study the ratio image [33], which is the ratio of the original image (speckled) by the denoised image. Ideally, in areas of the image where speckle is fully developed, this ratio should have the characteristics of pure speckle. Thus, the best filter is the one for which the ratio image has the mean value closer to unity and the equivalent number of looks closer to 4 . The results of this experiment are shown in Table 2 together with the results obtained after processing the original image by means of Kuan, Frost, ГMAP and MBD [33] filters.

The results of the above experiment seem to be consistent with the simulation results in the previous section, the best performance being again achieved by our proposed algorithm. Altough conceptually similar with the ГMAP filter, we attribute the better performance of our proposed filter to the better performance of the generalized Rayleigh model in capturing the heavy-tailed nature of SAR data.

$\mathrm{RR} \mathrm{n}^{\circ} 5493$ 


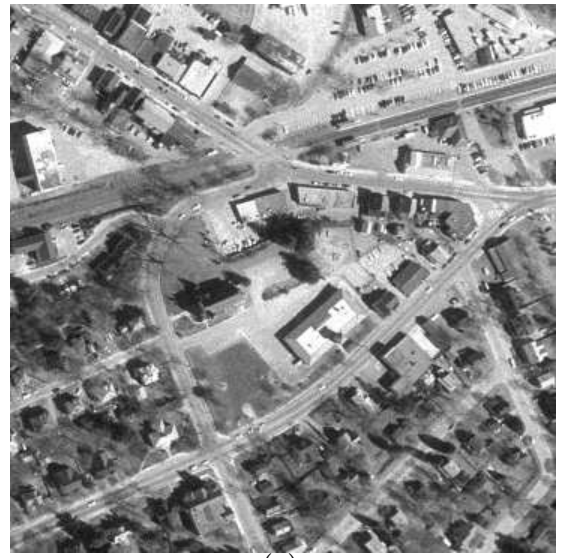

(a)

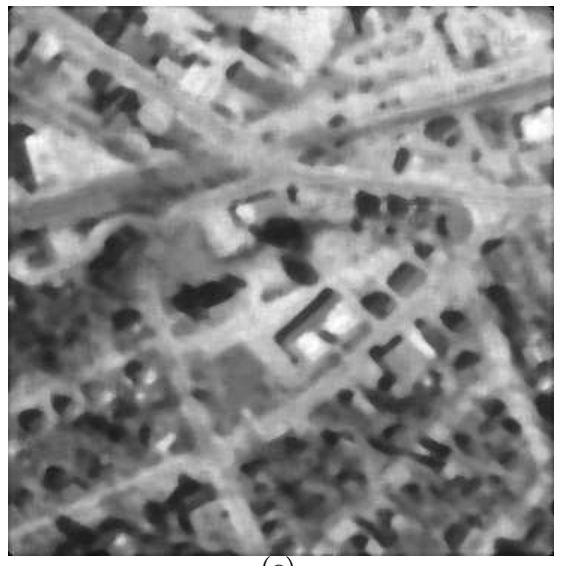

(c)

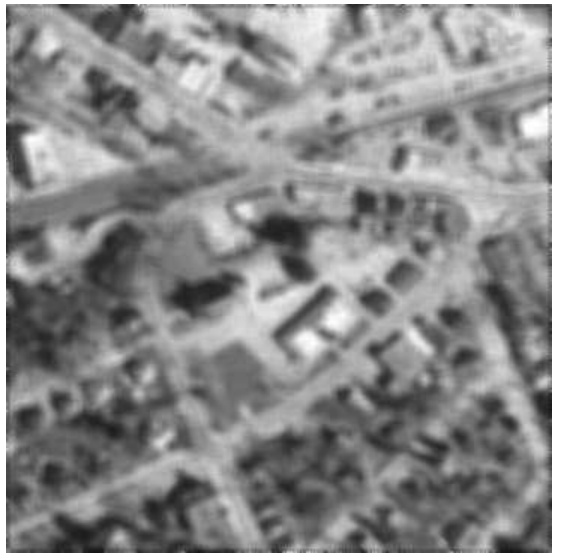

(e)

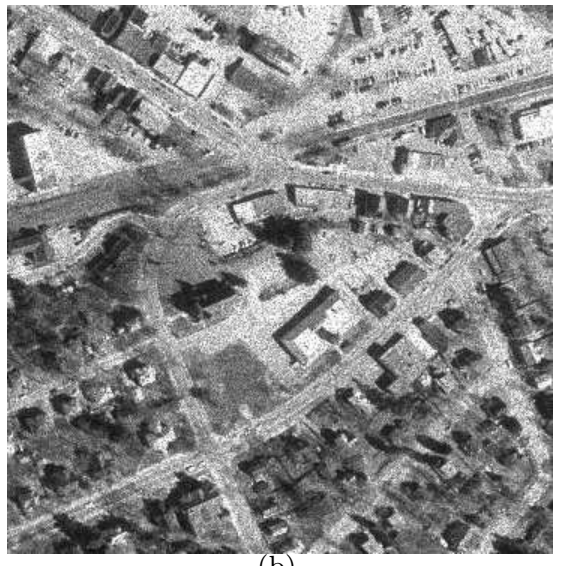

(b)

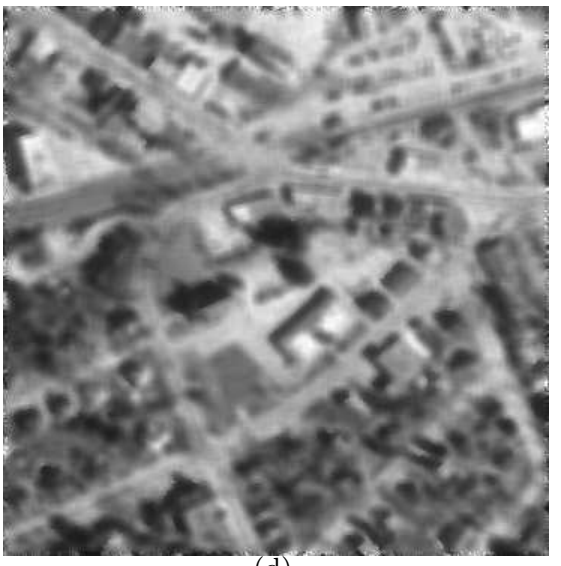

(d)

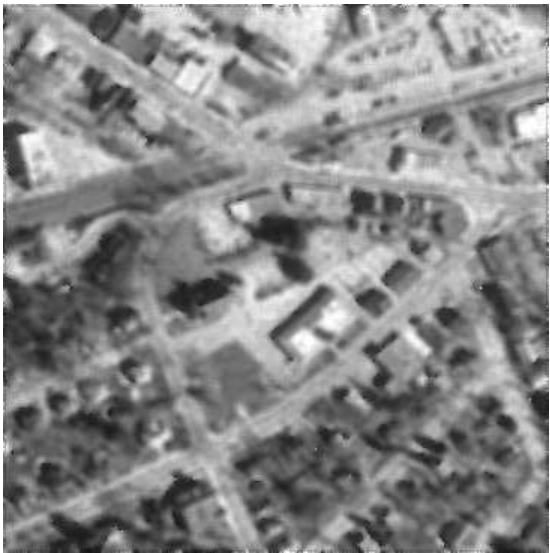

(f)

Figure 2: Results of various despeckling methods. (a) Original aerial image. (b) Image degraded with simulated speckle noise ( $L=3$, amplitude format). (c) Median filter. (d) Homomorphic Wiener filter. (e) Lee filter. (f) Proposed MAP filter based on the heavytailed Rayleigh model. 


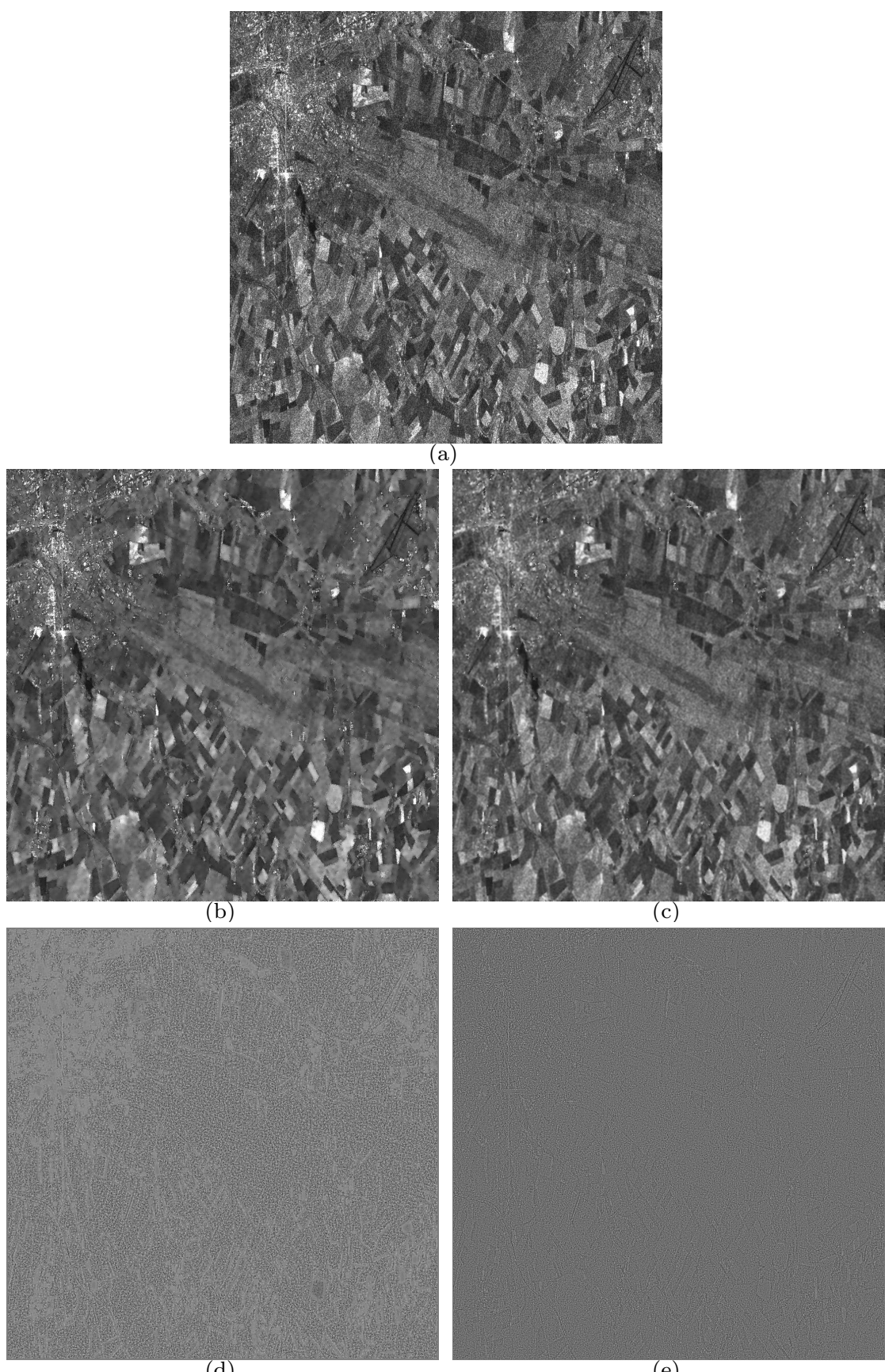

(d)

e)

Figure 3: (a) Original 4-look "Bourges" image. (b) ГMAP filtered (c) Proposed filter (d) Ratio image after $\Gamma M A P$ filtering (e) Ratio image after applying our algorithm 
Table 2: Quantitative filter evaluation for "Bourges" test image.

\begin{tabular}{|c||c|c|c|c|c|}
\hline Filter & Kuan & Frost & ГMAP & MBD & proposed \\
\hline \hline Mean & 0.94 & 0.96 & 1.05 & 0.98 & 0.99 \\
\hline ENL & 5.42 & 5.04 & 7.02 & 4.23 & 3.83 \\
\hline
\end{tabular}

\section{Conclusions}

We presented a new homomorphic statistical filter for speckle noise removal in SAR images, which is based on the recently introduced heavy-tailed Rayleigh model for the amplitude of the RCS. The model was developed based on the observation that the real and imaginary parts of the received complex signal can be accurately modelled using the symmetric alphastable family of distribution. Under the assumption of a multiplicative speckle noise model, we first employed a logarithmic transformation in order to change the noise into an additive one and to differentiate its characteristics from the signal characteristics. Then, a maximum a posteriori processor was implemented numerically and the corresponding nonlinearities were applied to the observed data. A novel parameter estimation method was developed for the case of generalized Rayleigh signal mixed with Gamma/Nakagami distributed speckle noise. The estimation method is based on the second-kind statistic theory employing Mellin's transform. We have applied our developed filter to a number of simulated as well as real speckle images and we compared the results with those obtained by means of classical speckle filters. Our simulations results showed that the homomorphic MAP filter based on the heavy-tailed Rayleigh model is among the best for speckle removal.

\section{Acknowledgement}

The authors would like to thank the French Space Agency CNES for providing the "Bourges" image available on the CD-ROM "Speckle filters comparative tests" (C) CNES, 2001). The first author would like to thank ERCIM (European Research Consortium in Informatics and Mathematics) for partial financial support during his post-doc at ISTI-CNR, Pisa, Italy and INRIA, Sophia Antipolis, France. 


\section{References}

[1] M. Soumekh, Synthetic Aperture Radar Signal Processing. New York: John Wiley and Sons, 1999.

[2] J. W. Goodman, "Some fundamental properties of speckle," J. Opt. Soc. Amer., vol. 66, pp. 1145-1150, November 1976.

[3] J. G. Abbott and F. L. Thurstone, "Acoustic speckle: Theory and experimental analysis," Ultrason. Imag., vol. 1, pp. 303-324, 1979.

[4] A. K. Jain, Fundamental of Digital Image Processing. NJ: Prentice-Hall, 1989.

[5] V. S. Frost, J. A. Stiles, K. S. Shanmugan, and J. C. Holtzman, "A model for radar images and its application to adaptive digital filtering of multiplicative noise," IEEE Trans. on Pattern Anal. and Machine Intell., vol. 4, pp. 157-166, 1982.

[6] D. T. Kuan, A. A. Sawchuk, T. C. Strand, and P. Chavel, "Adaptive noise smoothing filter for images with signal-dependent noise," IEEE Trans. on Pattern Anal. and Machine Intell., vol. 7, pp. 165-177, 1985.

[7] J. S. Lee, "Digital image enhancement and noise filtering by use of local statistics," IEEE Trans. on Pattern Anal. and Machine Intell., vol. 2, pp. 165-168, 1980.

[8] M. R. Azimi-Sadjadi and S. Bannour, "Two-dimensional adaptive block Kalman filtering of SAR imagery," IEEE Trans. on Geosci. and Remote Sensing, vol. 29, pp. 742-753, 1991.

[9] J. Bruniquel and A. Lopes, "Multi- variate optimal speckle reduction in SAR imagery," International Journal of Remote Sensing, vol. 18, pp. 603-627, Feb 1997.

[10] A. Baraldi and F. Parmigiani, "A refined Gamma MAP SAR speckle filter with improved geometrical adaptivity," IEEE Trans. on Geosci. and Remote Sensing, vol. 33, pp. 12451257, Sept. 1995.

[11] F. Argenti and L. Alparone, "Speckle removal from SAR images in the undecimated wavelet domain," IEEE Trans. on Geosci. and Remote Sensing, vol. 40, pp. 2363-2374, Nov. 2002.

[12] H. Xie, L. E. Pierce, and F. T. Ulaby, "SAR speckle reduction using wavelet denoising and Markov random field modeling," IEEE Trans. on Geosci. and Remote Sensing, vol. 40, pp. 2196-2212, Oct. 2002.

$\mathrm{RR} \mathrm{n}^{\circ} 5493$ 
[13] A. Achim, P. Tsakalides, and A. Bezerianos, "SAR image denoising via Bayesian wavelet shrinkage based on heavy-tailed modeling," IEEE Trans. Geosci. and Remote Sensing, vol. 41, pp. 1773-1784, Aug. 2003.

[14] S. Solbo and T. Eltoft, "Hommomorphic wavelet-based statistical despeckling of SAR images," IEEE Trans. on Geosci. and Remote Sensing, vol. 42, pp. 711-721, April 2004.

[15] E. E. Kuruoglu and J. Zerubia, "Modeling SAR images with a generalization of the Rayleigh distribution," IEEE Trans. on Image Processing, vol. 13, pp. 527-533, April 2004 .

[16] C. L. Nikias and M. Shao, Signal Processing with Alpha-Stable Distributions and Applications. New York: John Wiley and Sons, 1995.

[17] P. Tsakalides and C. L. Nikias, "High-resolution autofocus techniques for SAR imaging based on fractional lower order statistics," IEE Proc.-Radar. Sonar Navig., vol. 148, pp. 267-276, Oct. 2001.

[18] G. Moser, J. Zerubia, and S. B. Serpico, "SAR amplitude probability density function estimation based on a generalized Gaussian scattering model," Research Report 5153, INRIA, March 2004.

[19] G. Moser, J. Zerubia, and S. B. Serpico, "Dictionary-based stochastic expectationmaximization for SAR amplitude probability density function estimation," Research Report 5154, INRIA, March 2004.

[20] J. Bertrand, P. Bertrand, and J. P. Ovarlez, "The mellin transform," in The Transforms and Applications Handbook (A. Poularikas, ed.), CRC Press, 1990.

[21] J. M. Nicolas, "Introduction aux statistiques de deuxième espèce: applications des logmoments et des log-cumulants à l'analyse des lois d'images radar," Traitement du Signal, vol. 19, pp. 139-167, 2002.

[22] C. Tison, J. M. Nicolas, F. Tupin, and H. Maitre, "A new statistical model for Markovian classification of urban areas in high resolution SAR images," IEEE Trans. on Geosci. and Remote Sensing, vol. 42, pp. 2046-2057, Oct. 2004.

[23] C. Oliver and S. Quegan, Understanding Synthetic Aperture Radar Images. Boston: Artech House, 1998. 
[24] A. C. Frery, H.-J. Müller, C. C. F. Yanasse, and S. J. S. Sant'Anna, "A model for extremely heterogeneous clutter," IEEE Trans. on Geoscience and Remote Sensing, vol. 35, pp. 648-659, May 1997.

[25] L. Gagnon and A. Jouan, "Speckle filtering of SAR images - a comparative study between complex-wavelet based and standard filters," SPIE Proc. \#3169, pp. 80-91, 1997.

[26] A. Achim, A. Bezerianos, and P. Tsakalides, "Novel Bayesian multiscale method for speckle removal in medical ultrasound images," IEEE Trans. Med. Imag., vol. 20, pp. 772-783, Aug. 2001.

[27] H. H. Arsenault and G. April, "Properties of speckle integrated with a finite aperture and logarithmically transformed," J. Opt. Soc. Amer., vol. 66, pp. 1160-1163, November 1976.

[28] H. Xie, L. E. Pierce, and F. T. Ulaby, "Statistical properties of logarithmically transformed speckle," IEEE Trans. on Geosci. and Remote Sensing, vol. 40, pp. 721-727, March 2002.

[29] B. Epstein, "Some applications of the Mellin transform in statistics," The Annals of Mathematical Statistics, vol. 19, pp. 370-379, Sep 1948.

[30] V. M. Zolotarev, "Mellin-Stieltjes transforms in probability theory," Theory of Probability and its Applications, no. 4, pp. 432-460, 1957.

[31] E. E. Kuruoglu and J. Zerubia, "Modeling SAR images with a generalization of the Rayleigh distribution," Proc. Asilomar Conference, Oct. 2000.

[32] F. Sattar, L. Floreby, G. Salomonsson, and B. Lövström, "Image enhancement based on a nonlinear multiscale method," IEEE Trans. Image Processing, vol. 6, pp. 888-895, June 1997.

[33] M. Walessa and M. Datcu, "Model-based despeckling and information extraction from SAR images," IEEE Trans. on Geosci. and Remote Sensing, vol. 38, pp. 2258-2269, Sep. 2000.

$\mathrm{RR} \mathrm{n}^{\circ} 5493$ 


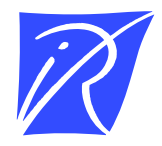

\section{Unité de recherche INRIA Sophia Antipolis 2004, route des Lucioles - BP 93 - 06902 Sophia Antipolis Cedex (France)}

Unité de recherche INRIA Futurs : Parc Club Orsay Université - ZAC des Vignes 4, rue Jacques Monod - 91893 ORSAY Cedex (France) Unité de recherche INRIA Lorraine : LORIA, Technopôle de Nancy-Brabois - Campus scientifique 615, rue du Jardin Botanique - BP 101 - 54602 Villers-lès-Nancy Cedex (France)

Unité de recherche INRIA Rennes : IRISA, Campus universitaire de Beaulieu - 35042 Rennes Cedex (France)

Unité de recherche INRIA Rhône-Alpes : 655, avenue de l'Europe - 38334 Montbonnot Saint-Ismier (France)

Unité de recherche INRIA Rocquencourt : Domaine de Voluceau - Rocquencourt - BP 105 - 78153 Le Chesnay Cedex (France) 